\title{
Applied Mathematical Sciences
}

\section{Volume 54}

Editors

F. John J. E. Marsden L. Sirovich

Advisors

H. Cabannes M. Ghil

J. K. Hale J. Keller G. B. Whitham 


\title{
Applied Mathematical Sciences
}

\author{
A Selection
}

21. Courant/Friedrichs: Supersonic Flow and Shock Waves.

22. Rouche/Habets/Laloy: Stability Theory by Liapunov's Direct Method.

23. Lamperti: Stochastic Processes: A Survey of the Mathematical Theory.

24. Grenander: Pattern Analysis: Lectures in Pattern Theory, Vol. II.

25. Davies: Integral Transforms and Their Applications, 2nd ed.

26. Kushner/Clark: Stochastic Approximation Methods for Constrained and Unconstrained Systems.

27. de Boor: A Practical Guide to Splines.

28. Keilson: Markov Chain Models-Rarity and Exponentiality.

29. de Veubeke: A Course in Elasticity.

30. Sniatycki: Geometric. Quantization and Quantum Mechanics.

31. Reid: Sturmian Theory for Ordinary Differential Equations.

32. Meis/Markowitz: Numerical Solution of Partial Differential Equations.

33. Grenander: Regular Structures: Lectures in Pattern Theory, Vol. III.

34. Kevorkian/Cole: Perturbation Methods in Applied Mathematics.

35. Carr: Applications of Centre Manifold Theory.

36. Bengtsson/Ghil/Källén: Dynamic Meterology: Data Assimilation Methods.

37. Saperstone: Semidynamical Systems in Infinite Dimensional Spaces.

38. Lichtenberg/Lieberman: Regular and Stochastic Motion.

39. Piccinini/Stampacchia/Vidossich: Ordinary Differential Equations in $\mathbf{R}^{\mathbf{n}}$.

40. Naylor/Sell: Linear Operator Theory in Engineering and Science.

41. Sparrow: The Lorenz Equations: Bifurcations, Chaos, and Strange Attractors.

42. Guckenheimer/Holmes: Nonlinear Oscillations, Dynamical Systems and Bifurcations of Vector Fields.

43. Ockendon/Tayler: Inviscid Fluid Flows.

44. Pazy: Semigroups of Linear Operators and Applications to Partial Differential Equations.

45. Glashoff/Gustafson: Linear Optimization and Approximation: An Introduction to the Theoretical Analysis and Numerical Treatment of Semi-Infinite Programs.

46. Wilcox: Scattering Theory for Diffraction Gratings.

47. Hale et al.: An Introduction to Infinite Dimensional Dynamical Systems - Geometric Theory.

48. Murray: Asymptotic Analysis.

49. Ladyzhenskaya: The Boundary-Value Problems of Mathematical Physics.

50. Wilcox: Sound Propagation in Stratified Fluids.

51. Golubitsky/Schaeffer: Bifurcation and Groups in Bifurcation Theory, Vol. I.

52. Chipot: Variational Inequalities and Flow in Porous Media.

53. Majda: Compressible Fluid Flow and Systems of Conservation Laws in Several Space Variables.

54. Wasow: Linear Turning Point Theory.

55. Yosida: Operational Calculus: A Theory of Hyperfunctions.

56. Chang/Howes: Nonlinear Singular Perturbation Phenomena: Theory and Applications. 
Wolfgang Wasow

\section{Linear Turning Point Theory}

With 19 Illustrations

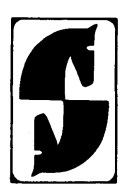

Springer Science+Business Media, LLC 


\author{
Wolfgang Wasow \\ University of Wisconsin-Madison \\ Mathematics Department \\ Madison, Wisconsin 53706 \\ U.S.A.
}

\title{
Editors
}

\begin{tabular}{|c|c|c|}
\hline F. John & J. E. Marsden & L. Sirovich \\
\hline $\begin{array}{l}\text { Courant Institute of } \\
\text { Mathematical Sciences }\end{array}$ & $\begin{array}{l}\text { Department of } \\
\text { Mathematics }\end{array}$ & $\begin{array}{l}\text { Division of } \\
\text { Applied Mathematics }\end{array}$ \\
\hline New York University & University of California & Brown University \\
\hline New York, NY 10012 & Berkeley, CA 94720 & Providence, RI 02912 \\
\hline U.S.A. & U.S.A. & U.S.A. \\
\hline
\end{tabular}

AMS Subject Classifications: 65L99, 34E99, 34A30, 34A50

\section{Library of Congress Cataloging in Publication Data}

Wasow, Wolfgang Richard,

Linear turning point theory.

(Applied mathematical sciences; vol. 54)

Bibliography: $p$.

1. Differential equations-Asymptotic theory.

I. Title. II. Series: Applied mathematical sciences

(Springer-Verlag New York Inc.); v. 54.

$\begin{array}{llll}\text { QA1.A647 } & \text { vol. } 54 & 510 \mathrm{~s} & 84-20243 \\ \text { [QA372] } & & {\left[513.3^{\prime} 5\right]} & \end{array}$

ISBN 978-1-4612-7008-9 ISBN 978-1-4612-1090-0 (eBook)

DOI 10.1007/978-1-4612-1090-0

(C) 1985 by Springer Science+Business Media New York

Originally published by Springer-Verlag New York Inc. in 1985

Softcover reprint of the hardcover 1st edition 1985

All rights reserved. No part of this book may be translated or reproduced in any form without written permission from Springer Science+Business Media, LLC.

Typeset by Computype, Inc., St. Paul, Minnesota. 


\section{Preface}

My book "Asymptotic Expansions for Ordinary Differential Equations" published in 1965 is out of print. In the almost 20 years since then, the subject has grown so much in breadth and in depth that an account of the present state of knowledge of all the topics discussed there could not be fitted into one volume without resorting to an excessively terse style of writing.

Instead of undertaking such a task, I have concentrated, in this exposition, on the aspects of the asymptotic theory with which I have been particularly concerned during those 20 years, which is the nature and structure of turning points. As in Chapter VIII of my previous book, only linear analytic differential equations are considered, but the inclusion of important new ideas and results, as well as the development of the necessary background material have made this an exposition of book length.

The formal theory of linear analytic differential equations without a parameter near singularities with respect to the independent variable has, in recent years, been greatly deepened by bringing to it methods of modern algebra and topology. It is very probable that many of these ideas could also be applied to the problems concerning singularities with respect to a parameter, and I hope that this will be done in the near future. It is less likely, however, that the analytic, as opposed to the formal, aspects of turning point theory will greatly benefit from such an algebraization. Also, mathematically trained readers who are mostly interested in applications may prefer the more elementary, narrative style in which my previous book was written, and which I have accepted for the present one as well.

The book is primarily concerned with general mathematical methods, but its contents may help applied mathematicians to understand better the many special cases, modifications and additions that can be found in the extensive literature. 
The presupposed mathematical background includes the elements of advanced calculus, complex variable theory, linear algebra, and ordinary differential equations, but no knowledge that is not normally acquired by an undergraduate student of mathematics whose main mathematical interest is Analysis.

I am grateful to the Mathematics Department of the University of Wisconsin-Madison, from which I retired four years ago, for allowing me to draw freely on its secretarial services in preparing the manuscript of this book, and special thanks are due to its capable and patient staff of technical typists.

Wolfgang Wasow 


\section{Contents}

Preface $v$

Chapter I Historical Introduction

1.1. Early Asymptotic Theory Without Turning Points 1

1.2. Total Reflection and Turning Points 3

1.3. Hydrodynamic Stability and Turning Points 5

1.4. The So-Called WKB Method 7

1.5. The Contribution of R. E. Langer 8

1.6. Remarks on Recent Trends 9

Chapter II Formal Solutions

2.1. Introduction 11

2.2. The Jordan Form of Holomorphic Functions 13

2.3. A Formal Block Diagonalization 16

2.4. Parameter Shearing: Its Nature and Purpose 22

2.5. Simplification by a Theorem of Arnold 24

2.6. Parameter Shearing: Its Application 26

2.7. Parameter Shearing: The Exceptional Case 29

2.8. Formal Solution of the Differential Equation 31

2.9. Some Comments and Warnings 34

Chapter III Solutions Away From Turning Points

3.1. Asymptotic Power Series: Definition of Turning Points 37

3.2. A Method for Proving the Analytic Validity of Formal
Solutions: Preliminaries 
3.3. A General Theorem on the Analytic Validity of Formal Solutions

3.4. A Local Asymptotic Validity Theorem 44

3.5. Remarks on Points That Are Not Asymptotically Simple 48

Chapter IV Asymptotic Transformations of Differential Equations

4.1. Asymptotic Equivalence 51

4.2. Formal Invariants 54

4.3. Formal Circuit Relations with Respect to the Parameter 58

Chapter V Uniform Transformations at Turning Points: Formal Theory

5.1. Preparatory Simplifications

5.2. A Method for Formal Simplification in Neighborhoods of a Turning Point

5.3. The Case $h>1$

5.4. The General Theory for $n=2$

Chapter VI Uniform Transformations at Turning Points: Analytic Theory

6.1. Preliminary General Results

6.2. Differential Equations Reducible to Airy's Equation 91

6.3. Differential Equations Reducible to Weber's Equation 98

6.4. Uniform Transformations in a Full Neighborhood of a Turning Point

6.5. Complete Reduction to Airy's Equation 104

6.6. Reduction to Weber's Equation in Wider Sectors 109

6.7. Reduction to Weber's Equation in a Full Disk 116

Chapter VII Extensions of the Regions of Validity of the Asymptotic Solutions

7.1. Introduction

7.2. Regions of Asymptotic Validity Bounded by Separation Curves: The Problem

7.3. Solutions Asymptotically Known in Sectors Bounded by Separation Curves

7.4. Singularities of Formal Solutions at a Turning Point 128

7.5. Asymptotic Expansions in Growing Domains

7.6. Asymptotic Solutions in Expanding Regions:

A General Theorem

7.7. Asymptotic Solutions in Expanding Regions:

A Local Theorem 
Chapter VIII Connection Problems

$\begin{array}{ll}\text { 8.1. Introduction } & 140\end{array}$

8.2. Stretching and Parameter Shearing 142

8.3. Calculation of the Restraint Index 146

8.4. Inner and Outer Solutions for a Particular $n$ th-Order System $\quad 150$

8.5. Calculation of a Central Connection Matrix 155

8.6. Connection Formulas Calculated Through

Uniform Simplification

Chapter IX Fedoryuk's Global Theory of Second-Order Equations

9.1. Global Formal Solutions of $\epsilon^{2} u^{\prime \prime}=a(x) u$

9.2. Separation Curves for $\epsilon^{2} u^{\prime \prime}=a(x) u$

9.3. A Global Asymptotic Existence Theorem for $\epsilon^{2} u^{\prime \prime}=a(x) u$

Chapter X Doubly Asymptotic Expansions

10.1. Introduction

180

10.2. Formal Solutions for Large Values of the Independent Variable

10.3. Asymptotic Solutions for Large Values of the Independent Variable

10.4. Some Properties of Doubly Asymptotic Solutions 190

10.5. Central Connection Problems in Unbounded Regions 194

Chapter XI A Singularly Perturbed Turning Point Problem

11.1. The Problem

11.2. A Simple Example 199

11.3. The General Case: Formal Part 204

11.4. The General Case: Analytic Part 208

Chapter XII Appendix: Some Linear Algebra for Holomorphic Matrices

12.1. Vectors and Matrices of Holomorphic Functions 215

12.2. Reduction to Jordan Form 221

12.3. General Holomorphic Block Diagonalization 226

12.4. Holomorphic Transformation of Matrices into Arnold's Form $\quad 228$

$\begin{array}{ll}\text { References } & 237\end{array}$

Index 